\section{Cytokinin and Ethephon Affect Crop Load, Shoot Growth, and Nutrient Concentration of 'Empire' Apple Trees}

\author{
D.C. Elfving ${ }^{1}$ and R.A. Cline ${ }^{2}$ \\ Horticultural Research Institute of Ontario, Box 7000, Vineland Station, Ont. \\ LOR 2EO, Canada
}

Additional index words. benzyladenine, thidiazuron, chemical thinning, fruit size, yield, vegetative growth, Malus domestica

Abstract. Benzyladenine (BA) applied postbloom at 125 and 250 mg-liter' ${ }^{-1}$ thinned 'Empire' apple (Malus domestica Borkh.) trees below commercial crop levels but thinned less than thidiazuron (THI) at 62 and $125 \mathrm{mg} \cdot \mathrm{liter}^{-1}$. Ethephon (ETH) applied up to 250 mg-liter ${ }^{-1}$ reduced fruit set only slightly. When BA was tank-mixed with ETH, thinning was the same as with BA alone. Although THI thinned more, BA resulted in a larger increase in fruit weight. Seed development was nearly eliminated by THI, but was unaffected by either BA or ETH. Thinner effects on foliar nutrient concentrations were associated with changes in fruit load but not shoot growth. The effects of BA and ETH on fruit-flesh nutrient concentrations were similar to their effects on foliar nutrient concentrations. Although THI thinned strongly and produced large changes in foliar nutrient concentrations and seed count, THI resulted in virtually no changes in fruit-flesh nutrient concentrations. Chemical names used: $\mathrm{N}$ - (phenylmethyl)-1 $\mathrm{H}$ - purine-6-amine (benzyladenine); 2-chlorophosphonic acid (ethephon); $N$ - phenyl- $N^{\prime}$ - 1,2,3-thiadiazol-5-ylurea (thidiazuron).

Chemically thinning apples postbloom improves fruit size, increases return bloom, and reduces cropping alternation (Forshey, 1987; Looney, 1986; Williams and Edgerton, 198 1). The 'Empire' apple, widely planted in Ontario, is noted for its moderate fruit size and increased difficulty in thinning older trees (Forshey, 1990). Ethephon (ETH) has shown thinning properties on several apple cultivars (Jones et al., 1983, 1989; Knight, 1980; Knight et rd., 1987; Keen et al., 1988). The cytokinins benzyladenine (BA) and $N$ - (2-chloro-4pyridyl) $-N^{\prime}$-phenylurea (CPPU) also thin apple (Bound et al., 1991; Byers and Carbaugh, 1991; Elfving, 1989; Elfving and Cline, 1993; Greene, 1989; Greene and Autio, 1989; Greene et al., 1990). Cytokinins and ethephon also can affect vegetative growth, nutrient concentrations, and crop load (Elfving, 1984; Miller, 1988). Thidiazuron (THI) has been reported to have cytokinin-like activity in apple and other species (Fellman et al., 1987; Wang et al., 1986), but has not been studied as a chemical thinner for apple. The trials reported here evaluated BA, ETH, and THI for effects on

Received for publication 8 Sept. 1992. Accepted for publication 4 Mar. 1993. Mention of a product or trade name does not constitute a guarantee or warranty of the product by the Horticultural Research Institute of Ontario nor an endorsement over similar products not mentioned. The cost of publishing this paper was defrayed in part by the payment of page charges. Under postal regulations, this paper therefore must be hereby marked advertisement solely to indicate this fact.

'Present address: Tree Fruit Research and Extension Center, Washington State Univ., 1100 N. Western Ave., Wenatchee, WA 98801.

${ }^{2}$ Research Scientist (retired). return bloom of 'Empire'/M.7 trees (Expt. 1).

yPCLA = per square centimeter of limb cross-sectional area. measured at a predetermined location at bloom, when shoot growth was measured in late August, and again the following spring. Trunk circumference was measured each year after leaf drop at a premarked location $30 \mathrm{~cm}$ above the soil surface.

Yield and mean fruit weight were determined by counting and weighing all fruit on each tree at harvest. In 1990, a 25-fruit sample was collected randomly from each tree at harvest. Fruit length and diameter were measured, and each fruit was sectioned equatorially to count normally developed seeds.

Midshoot leaves from current-season shoot growth were collected in Aug. 1989 and 1990 and analyzed according to the methods of Bodnar et al. (1983). Fruit samples were collected at harvest in 1990 and analyzed for nutrient concentrations using the same methods.

Experiment 1,1989: BA and ETH. Twelve treatments each were applied in a factorial treatment arrangement to a separate set of five-replicate, 8-year-old 'Empire'/M.7 trees in a randomized complete-block design. Each treatment consisted of a tank mix of one concentration of the following two growth regulators: 1) BA at $0,50,100$, or $150 \mathrm{mg}$ (a.i.)/liter and 2) ETH at 0, 50, or $100 \mathrm{mg}$ (a.i.)/liter. The control (0 BA + 0 ETH) was nontreated. Treatments were applied on 6 June $[13$ days after full bloom (DAFB)], when fruit diameter averaged $10.3 \pm 0.9 \mathrm{~mm}$. Trees were spaced 4.3 $\times 6.7 \mathrm{~m}$ on a moderately drained Tuscola silt loam consisting of mixed glaciolacustrine loams, silts, and very fine sandy loam (Hohner and Presant, 1989).

Experiment 2, 1990: BA, ETH, and THI. Seven treatments each were applied to sevenreplicate, 7-year-old 'Empire'/M.26 trees in a randomized complete-block design: 1) control (no treatment), 2) BA at 125 or $250 \mathrm{mg}$ (a.i.)/liter, 3) THI at 62 or $125 \mathrm{mg}$ (a.i.)/liter, or

Table 1. Effects of both BA and ETH on flowering, fruit set, yield, trunk cross-sectional area (TCSA), and

\begin{tabular}{|c|c|c|c|c|c|c|}
\hline \multirow[b]{2}{*}{$\begin{array}{l}\text { BA/ETH } \\
\left(\mathrm{mg} \cdot \text { liter }^{-1}\right)\end{array}$} & \multirow[b]{2}{*}{$\begin{array}{l}\text { Fruit } \\
\text { PCLA }^{y}\end{array}$} & \multicolumn{2}{|c|}{ Per tree } & \multirow{2}{*}{$\begin{array}{c}\text { Mean } \\
\text { fruit } \\
\text { wt } \\
(\mathrm{g}) \\
\end{array}$} & \multirow{2}{*}{$\begin{array}{c}\text { TCSA } \\
\text { increment, } \\
1989 \\
\left(\mathrm{~cm}^{2}\right)\end{array}$} & \multirow{2}{*}{$\begin{array}{c}\text { Blossom } \\
\text { clusters } \\
\text { PCLA, } \\
1990\end{array}$} \\
\hline & & $\begin{array}{l}\text { Fruit } \\
\text { (no.) }\end{array}$ & $\begin{array}{l}\text { Yield } \\
(\mathrm{kg})\end{array}$ & & & \\
\hline $0 / 0$ & 7.3 & 578 & 72 & 130 & 9.5 & 6.0 \\
\hline $50 / 0$ & 3.8 & 430 & 63 & 150 & 12.5 & 11.6 \\
\hline $100 / 0$ & 5.6 & 436 & 69 & 160 & 12.8 & 12.6 \\
\hline $150 / 0$ & 4.4 & 412 & 71 & 170 & 13.1 & 9.0 \\
\hline $0 / 50$ & 7.2 & 592 & 70 & 120 & 10.3 & 8.5 \\
\hline $50 / 50$ & 6.4 & 516 & 71 & 140 & 11.5 & 10.7 \\
\hline $100 / 50$ & 4.7 & 395 & 61 & 160 & 12.4 & 12.5 \\
\hline $150 / 50$ & 3.4 & 360 & 63 & 170 & 13.0 & 12.0 \\
\hline $0 / 100$ & 6.2 & 552 & 70 & 130 & 11.5 & 10.0 \\
\hline $50 / 100$ & 5.2 & 383 & 58 & 140 & 11.6 & 11.4 \\
\hline $100 / 100$ & 3.6 & 371 & 60 & 170 & 10.8 & 13.6 \\
\hline $150 / 100$ & 3.4 & 347 & 56 & 160 & 13.0 & 12.0 \\
\hline \multicolumn{7}{|l|}{ Significance } \\
\hline \multicolumn{7}{|l|}{$\mathrm{BA}$} \\
\hline $\mathrm{L}$ & $* * * *$ & $* * * *$ & NS & $* * * *$ & $*$ & * \\
\hline $\mathrm{Q}$ & NS & $*$ & NS & $*$ & NS & $*$ \\
\hline \multicolumn{7}{|l|}{ ETH } \\
\hline $\mathrm{L}$ & NS & NS & $*$ & NS & NS & * \\
\hline
\end{tabular}

${ }^{\mathrm{z}} 0 \mathrm{BA}+0$ ETH treatment was nontreated; each mean contains five observations.

Ns,*,****Nonsignificant or significant at $P \leq 0.05$ or 0.0001 , respectively; $\mathrm{L}=$ linear and $\mathrm{Q}=$ quadratic by multiple regression analysis. Higher-order simple and interactive effects nonsignificant $(P \leq 0.05)$. 
4) ETH at 125 or $250 \mathrm{mg}$ (a.i.)/liter. Treatments were applied on 31 May (22 DAFB), when fruit diameter averaged $11.0 \pm 2.5 \mathrm{~mm}$. Trees were spaced $3.7 \times 5.5 \mathrm{~m}$ on a welldrained Brant sandy loam consisting of a mixture of glaciolacustrine loams, silts, and fine sandy loam (Hohner and Presant, 1989).

Data from Expt. 1 were analyzed by multiple regression. Experiment 2 was analyzed using a radiating regression model described by Cochran and Cox (1957) and Elfving and Allen (1987). All analyses were carried out using the General Linear Models (GLM) procedure of the Statistical Analysis System (SAS) program package (SAS Institute, Cary, N.C.). In both experiments, quadratic or cubic regression models were analyzed initially, as appropriate. Nonsignificant higher-order effects were incorporated into the error term of less-complex models, which were reanalyzed.

Experiment 1. Flowering at the start of the trial was uniform among treatments, averaging 11.4 blossom clusters per square centimeter of limb cross-sectional area (PCLA) (data not shown). Fruit set and fruit count per tree were reduced by higher BA concentrations but were unaffected by ETH (Table 1). In contrast, yield per tree was reduced at higher ETH concentrations but was unaffected by BA. Mean fruit weight was increased substantially by BA but was not affected by ETH. Initial trunk cross-sectional area (TCSA) was uniform among treatments and averaged $58 \mathrm{~cm}^{2} /$ tree. Incremental trunk growth in 1989 increased with higher BA concentration, probably as a result of reduced crop load. Return bloom in 1990 was increased by BA and ETH.

Shoot count and length were increased and mean shoot length was decreased by BA, but shoot growth was unaffected by ETH (Table 2). Foliar $\mathrm{K}$ was increased and $\mathrm{Ca}$ decreased by $\mathrm{BA}$, while $\mathrm{N}$ and $\mathrm{Mg}$ were unaffected by either chemical.

Experiment 2. Flowering at the start of the trial was uniform among treatments, averaging 17 blossom clusters PCLA. Fruit set and fruit count per tree were decreased by a higher concentration of each chemical (Table 3). Yield per tree was decreased and mean fruit weight increased only by BA and THI. Return bloom in 1991 was increased only by BA and ETH. Initial TCSA was uniform among treatments and averaged $58 \mathrm{~cm}^{2} /$ tree. BA and THI increased incremental trunk enlargement in 1990 , probably because of reduced crop load.

Fruit length and diameter were increased by BA and THI, but ETH had no effect (Table 4). BA slightly decreased the length: diameter (LD) ratio. THI nearly eliminated seed development, similar to CPPU at $100 \mathrm{mg} \cdot \mathrm{liter}^{-1}$ on 'McIntosh' apples treated 18 days after petal fall (Greene, 1989), about the same time THI was applied in 1990. BA and ETH had no effect on seed count. Reduced seed count can influence fruit shape (Proctor and Schechter, 1992), but no such effects were observed in this study.

BA increased fruit-flesh $\mathrm{N}$ and $\mathrm{K}$ and decreased $\mathrm{P}$ and $\mathrm{Ca}$ concentrations (Table 4). The effect of BA on fruit-flesh Mg concentration was curvilinear. ETH had no effect on

Table 2. Effects of BA and ETH on shoot growth and foliar nutrient concentrations of 'Empire'/M.7 trees (Expt. 1).

\begin{tabular}{|c|c|c|c|c|c|c|c|}
\hline \multirow{3}{*}{$\begin{array}{l}\text { BA/ETH } \\
\left(\mathrm{mg} \cdot \text { liter }^{-1}\right)\end{array}$} & \multicolumn{2}{|c|}{$\begin{array}{c}\text { Shoot growth } \\
\text { PCLA }\end{array}$} & \multirow{3}{*}{$\begin{array}{l}\text { Mean } \\
\text { shoot } \\
\text { length } \\
(\mathrm{cm})\end{array}$} & \multirow{2}{*}{\multicolumn{4}{|c|}{ Foliar macronutrients ( $\%$ dry wt) }} \\
\hline & \multirow[b]{2}{*}{ No. } & \multirow{2}{*}{$\begin{array}{c}\text { Length } \\
(\mathrm{cm})\end{array}$} & & & & & \\
\hline & & & & $\mathrm{N}$ & $\mathrm{K}$ & $\mathrm{Ca}$ & $\overline{\mathrm{Mg}}$ \\
\hline$\overline{0 / 0}$ & 2.6 & 69 & 26 & 2.49 & 1.56 & 0.77 & 0.23 \\
\hline $50 / 0$ & 2.8 & 79 & 30 & 2.42 & 1.55 & 0.74 & 0.22 \\
\hline $100 / 0$ & 3.1 & 80 & 25 & 2.41 & 1.44 & 0.83 & 0.26 \\
\hline $150 / 0$ & 4.2 & 97 & 23 & 2.65 & 1.69 & 0.66 & 0.23 \\
\hline $0 / 50$ & 2.5 & 66 & 26 & 2.43 & 1.41 & 0.78 & 0.24 \\
\hline $50 / 50$ & 2.6 & 70 & 27 & 2.45 & 1.52 & 0.74 & 0.24 \\
\hline $100 / 50$ & 3.0 & 84 & 28 & 2.51 & 1.56 & 0.71 & 0.24 \\
\hline $150 / 50$ & 4.3 & 100 & 24 & 2.43 & 1.55 & 0.71 & 0.22 \\
\hline $0 / 100$ & 3.3 & 89 & 27 & 2.39 & 1.51 & 0.80 & 0.24 \\
\hline $50 / 100$ & 2.9 & 77 & 27 & 2.58 & 1.57 & 0.69 & 0.24 \\
\hline $100 / 100$ & 3.8 & 83 & 22 & 2.48 & 1.52 & 0.77 & 0.24 \\
\hline $150 / 100$ & 4.6 & 108 & 24 & 2.49 & 1.57 & 0.73 & 0.24 \\
\hline Significance & & & & & & & \\
\hline $\mathrm{BA}$ & & & & & & & \\
\hline $\mathrm{L}$ & $* * * *$ & $* *$ & $*$ & NS & $*$ & $*$ & NS \\
\hline$Q$ & * & NS & NS & NS & NS & NS & NS \\
\hline
\end{tabular}

${ }^{\text {xPCLA }}=$ per square centimeter of limb cross-sectional area.

$y_{0} \mathrm{BA}+0 \mathrm{ETH}$ treatment was nontreated; each mean contains five observations.

ss, ${ }^{* * *, * * * *}$ Nonsignificant or significant at $P \leq 0.05,0.01$, or 0.0001 , respectively; $\mathrm{L}=$ linear and $\mathrm{Q}=$ quadratic by multiple regression analysis. ETH effects and higher-order simple and interactive effects nonsignificant $(P \leq 0.05)$.

Table 3. Effects of BA, THI, or ETH on flowering, fruit set, yield, trunk cross-sectional area (TCSA), and return bloom of 'Empire'/M.26 trees (Expt. 2).

\begin{tabular}{|c|c|c|c|c|c|c|}
\hline \multirow[b]{2}{*}{$\begin{array}{l}\text { Treatment } \\
\left(\text { mg.liter }^{-1}\right)\end{array}$} & \multirow[b]{2}{*}{$\begin{array}{c}\text { Fruit } \\
\text { PCLA }^{y}\end{array}$} & \multicolumn{2}{|c|}{ Per tree } & \multirow{2}{*}{$\begin{array}{c}\text { Mean } \\
\text { fruit } \\
\text { wt } \\
(\mathrm{g}) \\
\end{array}$} & \multirow{2}{*}{$\begin{array}{c}\text { TCSA } \\
\text { increment, } \\
1990 \\
\left(\mathrm{~cm}^{2}\right)\end{array}$} & \multirow{2}{*}{$\begin{array}{c}\text { Blossom } \\
\text { clusters } \\
\text { PCLA, } \\
1991 \\
\end{array}$} \\
\hline & & $\begin{array}{l}\text { Fruit } \\
\text { (no.) }\end{array}$ & $\begin{array}{l}\text { Yicld } \\
(\mathrm{kg})\end{array}$ & & & \\
\hline Control & 8.1 & 785 & 123 & 160 & 11.0 & 9.6 \\
\hline \multicolumn{7}{|l|}{$\mathrm{BA}$} \\
\hline 125 & 3.3 & 390 & 81 & 210 & 17.2 & 17.5 \\
\hline 250 & 1.6 & 225 & 56 & 250 & 22.5 & 22.7 \\
\hline \multicolumn{7}{|l|}{ THI } \\
\hline 62 & 2.8 & 227 & 46 & 230 & 18.7 & 14.8 \\
\hline 125 & 0.8 & 125 & 29 & 230 & 20.8 & 13.1 \\
\hline \multicolumn{7}{|l|}{ ETH } \\
\hline 125 & 5.8 & 625 & 106 & 170 & 13.6 & 13.8 \\
\hline 250 & 4.9 & 543 & 96 & 180 & 15.0 & 15.0 \\
\hline \multicolumn{7}{|l|}{ Significance } \\
\hline Concentration (C) & $* * * *$ & $* * * *$ & $* * * *$ & $* * * *$ & $* *$ & $* * *$ \\
\hline Growth regulator $\times C$ & $* * * *$ & $* * * *$ & $* * * *$ & $* * * *$ & $*$ & $* * *$ \\
\hline $\mathrm{BA}$ & $\mathrm{L}^{* * * *}$ & $\mathrm{~L}^{* * *}, \mathrm{Q}^{*}$ & $\mathrm{~L}^{* * * * *}$ & $\mathbf{L}^{* * * *}$ & $\mathrm{~L}^{* * *}$ & $\mathrm{~L}^{* * * * *}$ \\
\hline THI & $\mathrm{L}^{* * * *}$ & $\mathrm{~L}^{* * * *}, \mathrm{Q}^{* * * *}$ & $\mathrm{~L}^{* * * *}, \mathrm{Q}^{* * *}$ & $\mathrm{~L}^{* * * *}, \mathrm{Q}^{* * * *}$ & $\mathbf{L}^{* *}$ & NS \\
\hline ETH & $\mathbf{L}^{*}$ & $\mathrm{~L}^{*}$ & NS & NS & NS & $\mathrm{L}^{*}$ \\
\hline
\end{tabular}

${ }^{\mathrm{z}}$ Each mean contains seven observations.

yCLA = per square centimeter of limb cross-sectional area.

${ }_{\mathrm{NS},+, * *,+* *,+*+*}$ Nonsignificant or significant at $P \leq 0.05,0.01,0.001$, or 0.0001 , respectively; $\mathrm{L}=$ linear and $\mathrm{Q}$ $=$ quadratic by radiating-regression analysis.

fruit-flesh $\mathrm{N}$; effects similar to those of $\mathrm{BA}$ on fruit-flesh $\mathrm{P}, \mathrm{K}$, and $\mathrm{Mg}$; and a curvilinear effect on fruit-flesh $\mathrm{Ca}$ concentration. THI effects on fruit-flesh nutrient concentrations were limited to an increase in fruit-flesh $\mathrm{K}$ concentration.

BA increased shoot count and length but did not affect mean shoot length (Table 5). THI and ETH did not affect shoot count or length, but both reduced mean shoot length similarly. THI significantly decreased foliar $\mathrm{N}, \mathrm{K}, \mathrm{Ca}$, and $\mathrm{Mg}$ concentrations. Higher BA concentrations were accompanied by increased foliar $\mathrm{K}$ and decreased foliar $\mathrm{Ca}$ and $\mathrm{Mg}$ concentrations, but ETH did not influence foliar nutrient concentrations.

BA reduced 'Empire' fruit set in direct proportion to concentration up to $250 \mathrm{mg} \cdot \mathrm{liter}^{-1}$ with no negative effects on fruit size, shape, or seed development. Previous work with 'Empire' apple suggests that BA concentrations of 50 to $100 \mathrm{mg} \cdot$ liter $^{-1}$ thin adequately (Elfving and Cline, 1993). ETH thinning on 'Empire' trees was limited at concentrations up to 250 mg.liter ${ }^{-1}$. Jones et al. $(1989,1991)$ reported acceptable thinning of 'Fuji' and 'Delicious' with ETH at 25 to $200 \mathrm{mg} \cdot \mathrm{liter}^{-1}$, but other reports suggest that higher concentrations are necessary to reduce crop load significantly (Jones et al., 1983; Keen and Jones, 1985). Tank-mixing ETH with BA did not change BA thinning behavior or produce interactive effects on vegetative growth or crop load. THI was much more active as a thinner than BA and nearly defruited trees treated with 125 $\mathrm{mg} \cdot$ liter $^{-1}$. 
Table 4. Effects of BA, THI, or ETH on fruit characteristics and fruit-flesh nutrient concentrations of 'Empire'/M.26 trees (Expt. 2).

\begin{tabular}{|c|c|c|c|c|c|c|c|c|c|}
\hline \multirow{3}{*}{$\begin{array}{l}\text { Treatment }^{x} \\
\text { (mg.liter }{ }^{-1} \text { ) }\end{array}$} & \multicolumn{3}{|c|}{ Fruit } & \multirow{3}{*}{$\begin{array}{c}\text { Seeds/ } \\
\text { fruit } \\
\text { (no.) }\end{array}$} & \multirow{2}{*}{\multicolumn{5}{|c|}{$\begin{array}{l}\text { Fruit-flesh nutrient concn } \\
(\mathrm{mg} / 100 \mathrm{~g} \text { fresh } \mathrm{wt})\end{array}$}} \\
\hline & \multirow{2}{*}{$\begin{array}{l}\text { Length } \\
(\mathrm{cm})\end{array}$} & \multirow{2}{*}{$\begin{array}{l}\text { Diam } \\
(\mathrm{cm})\end{array}$} & \multirow{2}{*}{$\begin{array}{l}\overline{\mathrm{L}: \mathrm{D}^{y}} \\
\text { ratio }\end{array}$} & & & & & & \\
\hline & & & & & $\mathrm{N}$ & $P$ & $\mathrm{~K}$ & $\mathrm{Ca}$ & $\mathrm{Mg}$ \\
\hline $\begin{array}{l}\text { Control } \\
\text { BA }\end{array}$ & 6.5 & 7.2 & 0.90 & .5 .5 & 35 & $8 . \overline{2}$ & 64 & 5.1 & 4.2 \\
\hline 125 & 6.7 & 7.9 & 0.86 & 5.6 & 32 & 7.8 & 68 & 4.1 & 3.9 \\
\hline 250 & 7.3 & 8.2 & 0.89 & 4.9 & 40 & 5.4 & 110 & 4.6 & 4.4 \\
\hline THI & & & & & & & & & \\
\hline 62 & 7.2 & 8.0 & 0.91 & 0.9 & 31 & 7.9 & 83 & 4.3 & 4.3 \\
\hline 125 & 7.3 & 8.0 & 0.91 & 0.6 & 37 & 8.9 & 117 & 4.2 & 4.5 \\
\hline ETH & & & & & & & & & \\
\hline $125^{\circ}$ & 6.5 & 7.4 & 0.87 & 5.3 & 32 & 7.7 & 74 & 3.8 & 3.8 \\
\hline 250 & 6.3 & 7.3 & 0.86 & 5.7 & 38 & 6.8 & 96 & 5.6 & 4.7 \\
\hline Significance & & & & & & & & & \\
\hline Concn $(C)$ & $* * *$ & $* * * *$ & NS & $* * * *$ & $*$ & * & $* * * *$ & ** & ** \\
\hline $\mathrm{GR}^{\mathrm{x}} \times \mathrm{C}$ & $* * * *$ & $* * * *$ & $* *$ & $* * * *$ & NS & $* *$ & * & * & * \\
\hline BA & $\mathrm{L}^{* * * *}$ & $\mathrm{~L}^{* * * *}$ & $L^{*}, Q^{*}$ & NS & $\mathrm{L}^{*}$ & $\mathrm{~L}^{* *}$ & $\mathrm{~L}^{* * * * *}$ & $L^{*}$ & $Q^{*}$ \\
\hline THI & $\mathrm{L}^{* * *}, \mathrm{Q}^{*}$ & $\mathrm{~L}^{* * *}, \mathrm{Q}^{*}$ & NS & $\mathrm{L}^{* * * *}, \mathrm{Q}^{* * * *}$ & NS & NS & $L^{* * * * *}$ & NS & NS \\
\hline ETH & NS & NS & NS & NS & NS & $\mathbf{L}^{*}$ & $L^{* * * *}$ & $\mathrm{~L}^{* *}, \mathrm{Q}^{* * *}$ & $\mathrm{~L}^{* *}, \mathrm{Q}^{* *}$ \\
\hline
\end{tabular}

${ }^{\mathrm{z}}$ Each mean contains seven observations.

${ }^{y} \mathrm{~L}: \mathrm{D}=$ length : diameter ratio.

${ }^{\mathrm{x}} \mathrm{GR}=$ growth regulator.

Ns,*******,****Nonsignificant or significant at $P \leq 0.05,0.01,0.001$, or 0.0001 , respectively; $\mathrm{L}=$ linear and $\mathrm{Q}$ $=$ quadratic by radiating-regression analysis.

Table 5. Effects of BA, THI, or ETH on shoot growth and foliar nutrient concentrations of 'Empire'/M.26 trees (Expt. 2).

\begin{tabular}{|c|c|c|c|c|c|c|c|}
\hline \multirow{3}{*}{$\begin{array}{l}\text { Treatment }{ }^{y} \\
\left(\mathrm{mg} \cdot \text { liter }^{-1}\right)\end{array}$} & \multicolumn{2}{|c|}{$\begin{array}{l}\text { Shoot growth } \\
\text { PCLA }^{z}\end{array}$} & \multirow{3}{*}{$\begin{array}{c}\text { Mean } \\
\text { shoot } \\
\text { length } \\
(\mathrm{cm})\end{array}$} & \multirow{2}{*}{\multicolumn{4}{|c|}{ Foliar macronutrients (\% dry wt) }} \\
\hline & \multirow[b]{2}{*}{ No. } & \multirow{2}{*}{$\begin{array}{l}\text { Length } \\
(\mathrm{cm})\end{array}$} & & & & & \\
\hline & & & & $\mathrm{N}$ & $\mathrm{K}$ & $\mathrm{Ca}$ & $\mathrm{Mg}$ \\
\hline Control & 3.0 & 70 & 24 & 2.63 & 1.29 & 1.09 & 0.31 \\
\hline BA & & & & & & & \\
\hline 125 & 3.9 & 97 & 24 & 2.58 & 1.48 & 1.00 & 0.28 \\
\hline 250 & 5.2 & 104 & 19 & 2.57 & 1.64 & 0.90 & 0.27 \\
\hline THI & & & & & & & \\
\hline 62 & 2.7 & 55 & 19 & 2.11 & 1.77 & 0.82 & 0.24 \\
\hline 125 & 2.5 & 50 & 21 & 2.10 & 1.90 & 0.85 & 0.24 \\
\hline ETH & & & & & & & \\
\hline 125 & 2.7 & 56 & 21 & 2.59 & 1.35 & 1.10 & 0.30 \\
\hline 250 & 3.0 & 57 & 19 & 2.54 & 1.34 & 1.12 & 0.29 \\
\hline Significance & & & & & & & \\
\hline Concentration (C) & * & NS & * & $* * *$ & $* * *$ & $* *$ & * \\
\hline Growth regulator $\times C$ & $* * * *$ & $* * * *$ & NS & $* * * *$ & $* * * *$ & $* * * *$ & ** \\
\hline BA & 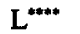 & $\mathrm{L}^{* *}$ & $\mathrm{~L}^{*}$ & NS & $\mathrm{L}^{* *}$ & $\mathrm{~L}^{* *}$ & $\mathrm{~L}^{*}$ \\
\hline THI & NS & Ns & $\mathrm{L}^{*}$ & $\mathrm{~L}^{* * * *}, \mathrm{Q}^{* * * *}$ & $L^{* *}, Q^{*}$ & $\mathbf{L}^{* * * *}, \mathrm{Q}^{* * * *}$ & $L^{* *}, Q^{*}$ \\
\hline ETH & NS & NS & $\mathrm{L}^{*}$ & NS & NS & NS & NS \\
\hline
\end{tabular}

${ }^{2} \mathrm{PCLA}=$ per square centimeter of limb cross-sectional area.

'Each mean contains seven observations.

ss, ,**********Nonsignificant or significant at $P \leq 0.05,0.01,0.001$, or 0.0001 , respectively; $\mathrm{L}=$ linear and $\mathrm{Q}$ $=$ quadratic by radiating-regression analysis.

High-volume applications, such as those used in the trials reported here, favor the maximum thinning response to ETH (Jones et al., 1991). Jones and Keen (1985) reported a linear increase in ETH-induced thinning from 12 to $24 \mathrm{C}$. The daily temperatures during the week after applying ETH in 1989 and 1990 averaged 18 and $16 \mathrm{C}$, respectively. Ambient temperatures may have limited ETH thinning.

BA increased shoot growth in both experiments, as has been observed previously (Bound et al., 1991; Elfving and Cline, 1993; Greene and Autio, 1990; Greene et al., 1990). ETH has reduced shoot growth when applied at $>350$ $\mathrm{mg} \cdot \mathrm{liter}^{-1}$ for thinning postbloom (Jones et al., 1983; Keen et al., 1988) but had little effect in these trials. Although THI exhibits cytokininlike activity and thinned more than BA, THI did not increase shoot count but reduced mean nutrient concentrations similarly to foliar nutrient concentrations. Despite its strong influence on foliar nutrient concentrations and seed count, THI had almost no effect on fruit-flesh nutrient concentrations. Bramlage et al. (1990) reported a direct relation between seed count at harvest and fruit size or flesh $\mathrm{Ca}$ concentration in 'Delicious' apple. There was no relationship between seed count and fruit size or fruit-flesh concentration of any nutrient determined in this study.

The risk of overthinning 'Empire' apple trees with BA is limited by the wide range of concentrations over which thinning remains inversely proportional to concentration. Successfully thinning 'Empire' apples with ETH may require significantly higher concentrations and changes in application timing than used here (Keen and Jones, 1985). The excessive thinning, reduced foliar $\mathrm{N}$ concentration, and detrimental effects on seed development observed with THI in this study may be ameliorated by using lower concentrations.

\section{Literature Cited}

Bodnar, J.M., J.T.A. Proctor, J.E. Laing, and R.A. Cline. 1983. Nutrient changes in apple leaves due to the spotted tentiform leaf miner. J. Amer. Soc. Hort. Sci. 108:685-688.

Bound, S.A., K.M. Jones, T.B. Keen, and M.J. Oakford. 1991. The thinning effect of benzyladenine on red 'Fuji' apple trees. J. Hort. Sci. 66:789-794.

Bramlage, W. J., S.A. Weis, and D.W. Greene. 1990. Observations on the relationships among seed number, fruit calcium, and senescent breakdown in apples. HortScience 25:351-353.

Byers, R.E. and D.H. Carbaugh. 1991. Effect of chemical thinning sprays on apple fruit set. HortTechnology 1:41-48.

Cochran, W.G. and G.M. Cox. 1957. Experimental designs, 2nd ed. section 3.43. Wiley, New York. p. $65-70$.

Elfving, D.C. 1984. Factors affecting apple tree response to chemical branch-induction treatments. J. Amer. Soc. Hort. Sci. 109:476-481.

Elfving, D.C. 1989. $N$ - (phenylmethyl) -1 $H$ - purine6-amine (BA) as a chemical thinner for 'Idared' apple. Acta Hort. 239:357-362.

Elfving, D.C. and O.B. Allen. 1987. Effect of gibberellin $\mathrm{A}_{4+7}$, applications on 'Golden Delicious' fruit russet. Crop Res. 27: 11-18.

Elfving, D.C. and R.A. Cline. 1993. Benzyladenine and other chemicals for thinning 'Empire' apple trees. J. Amer. Soc. Hort. Sci. 118:593-598.

Fellman, C.D., P.E. Read, and M.A. Hosier. 1987. Effects of thidiazuron and CPPU on meristem formation and shoot proliferation. HortScience 22:1197-1200.

Forshey, C.G. 1987. A review of chemical fruit thinning. Proc. N.E. Fruit Meetings 93:68-73.

Forshey, C.G. 1990. Factors affecting 'Empire' fruit size. Proc. New York State Hort. Soc. 135:7174

Forshey, C.G. and D.C. Elfving. 1979. Branch samples for yield and fruit size comparisons in apple. HortScience 14:143-144.

Greene, D.W. 1989. CPPU influences 'McIntosh' apple crop load and fruit characteristics. HortScience 24:94-96.

Greene, D.W. and W.R. Autio. 1989. Evaluation of benzyladenine as a chemical thinner on 'McIntosh' apples. J. Amer. Soc. Hort. Sci. 114:6873 .

Greene, D.W. and W.R. Autio. 1990. Vegetative 
responses of apple trees following benzyladenine and growth regulator sprays. J. Amer. Soc. Hort. Sci. 115:400-404.

Greene, D.W., W.R. Autio, and P. Miller. 1990. Thinning activity of benzyladenine on several apple cultivars. J. Amer. Soc. Hort. Sci. 115:394400.

Hohner, B. and T. Presant. 1989. Soils of the Horticultural Experiment Station Simcoe. Ont. Inst. of Pedology Publ. 89-3.

Jones, K.M. and T.B. Keen. 1985. Temperature effects on ethephon thinning of apples. J. Hort Sci. 60:21-24.

Jones, K.M., T.B. Keen, and R.J. Meredith. 1983. Thinning Golden Delicious apples using ethephon sprays. J. Hort. Sci. 58:381-388.

Jones, K.M., T.B. Keen, and M.J. Oakford. 1991 How volume of spray affects the thinning of red
'Delicious' with ethephon. N.Z. J. Crop Hort. Sci. 19:31-36.

Jones, K. M., T.B. Keen, M.J. Oakford, and S. Bound. 1989. Thinning 'Red Fuji' apples with ethephon or NAA. J. Hort. Sci. 64:527-532.

Knight, J.N. 1980. Fruit thinning of the apple cultivar Cox's Orange Pippin. J. Hort. Sci. 55:267273.

Knight, J.N., J.E. Spencer, N.E. Looney, and J.D. Lovell. 1987. Chemical thinning of the apple cultivar Spartan. J. Hort. Sci. 62:135-139.

Koen, T.B. and K.M. Jones. 1985. A model of ethephon thinning of Golden Delicious apples. J. Hort. Sci. 60:13-19.

Keen, T.B., K.M. Jones, and S.B. Longley. 1988. Spray thinning strategies for 'Red Delicious' apple using naphthalene acetic acid and ethephon. J. Hort. Sci. 63:31-35.
Looney, N.E. 1986. Chemical thinning of apples: Some new strategies and important refinements to old procedures. Acta Hort. 179:597-604.

Miller, S.S. 1988. Plant bioregulators in apple and pear culture. Hort. Rev. 10:309-401.

Proctor, J.T.A. and I. Schechter. 1992. Effect of ovule damage on fruit development in three apple cultivars. HortScience 27:18-19.

Snedecor, G.W. and W.G. Cochran. 1980. Statistical methods, 7th ed. section 17.5. Iowa State Univ. Press, Ames. p. 342-343.

Wang, S.Y., G.L. Steffens, and M. Faust. 1986. Breaking bud dormancy in apple with a plant bioregulator, thidiazuron. Photochemistry 25:311-317.

Williams, M.W. and L.J. Edgerton. 1981. Fruit thinning of apples and pears with chemicals. U.S. Dept. Agr., Agr. Info. Bu1. 289. 